\section{$\checkmark$ Research Square}

\title{
A Study on Brain-based Knowledge Model for Dentist's Clinical Decision Making
}

\section{Young-A Ji ( $\nabla_{\text {jyatree@gmail.com ) }}$}

Seoul National University School of Dentistry https://orcid.org/0000-0003-3202-7809

\section{Hong-Gee Kim}

The University of Sydney School of Dentistry

\section{Seungho Baek}

Seoul National University School of Dentistry

Jaeil Lee

Seoul National University School of Dentistry

\section{Research article}

Keywords: Clinical Decision making, EEG of Expert and Novice, Dentist's Knowledge Model, Brain-based learning

Posted Date: August 6th, 2020

DOI: https://doi.org/10.21203/rs.3.rs-44802/v1

License: (c) (1) This work is licensed under a Creative Commons Attribution 4.0 International License. Read Full License 


\section{Abstract \\ Background}

The expertise of dentists is very important since it improves the quality of dental services provided to patients. This study analyzed the characteristics of the decision-making process and confirmed dentists' electroencephalography(EEG) characteristics by measuring their cerebral activity during clinical decision making.

\section{Methods}

This study was performed in the following two projects: (1) A qualitative study of clinical decision making in a clinical scenario, (2) An analysis of differences in cortical activity of experts and novices through EEG.

\section{Results}

In the 1st study, this study found that 'confirmation of the patient's chief complaints', 'oral examination', and 'radiography reading' steps, which showed the most prominent differences in the experts' and novices' decision making, were set as the major steps of dentists' clinical decision making. In the 2nd study, the experts' and novices' cortical activities were measured through a 32-channel EEG. In task 6, which had the lowest accuracy of diagnoses made by the experts, the brain activities in both groups were higher than in other tasks.

\section{Conclusions}

This study aims to link learning and the cortical functions activated during dental experts' decision making in order to present learning directions for brain-based clinical decision making to develop experts.

\section{Background}

With social developments, societies have changed to knowledge-centered societies that require high levels of knowledge. This change has subsequently increased the importance of the role of experts who lead social changes and developments. In general, experts are defined as those who consistently demonstrate exceptional abilities in a certain area[1]. Dentists are also knowledge-oriented professionals who are always mentioned in the expert group. A study suggests that expertise requires systematic, highintensity training of at least 10 years or 10,000 hours. Such definition of experts can be inferred from the definition of expertise, which is often variably defined based on context. Expertise allows an individual to efficiently collect and manipulate information and to perform better in their area of expertise[2]. Many studies have sought to discover characteristics of expertise in experts. Ericsson estimated that 
professional musicians receive more than 10,000 hours of systematic training[3]. In other words, their research suggests that at least 10 years of intense training is required to achieve expertise[4].

Various studies have been conducted to analyze the characteristics of experts. In the expert performance approach, studies conduct experiments to objectively evaluate performance[5]. Here, the studies investigate the components of performance or comparatively analyze the differences between novices and experts to analyze the characteristics of experts[6].

Many studies have also investigated the components of expertise. In particular, knowledge, experience, and problem solving constitute the most basic components of generally accepted expertise[7]. Experts perform typical activities at each of the following stages: recognizing problems, defining problems, finding solutions, carrying out solutions, and reflection[8]. In experts, conceptually more abundant and organized representations occur, allowing them to solve given problems, and experts also tend to use abstract representation relying on in-depth knowledge. Here, knowledge representation refers to knowledge that externally expresses information organized within the cognitive framework[9]. Especially, clinical experts have a high knowledge structure, and based on this understanding, experts widen and deepen the representations while focusing on the fundamental principles[10]. Decision making, which refers to the process in which problems are recognized and solutions are selected upon consideration, is also defined as a process in which one reaches their selection among various possible alternatives through complex dental cognitive processes[11].

The brain consists of millions of neurons, and each neuron is connected to other neurons in various interrelationships. The interaction between these neurons, which can lead to learning, memory, behavior, decision making, and recognition, occur in synapses. Here, the information is transmitted in synapses through chemical substances, particularly due to the electric potential differences created by concentration gradients across the plasma membrane. Such electric potential differences were caused electric signals on the scalp, which are measured in EEG.

When compared to non-experts, experts have the ability to process unnecessary information without filtering and efficient neuronal networks[12]. Clinical experts can also make accurate selections in given tasks due to their abundant experiences with the tasks[13]. In addition, studies have also suggested that experts have higher activation of the frontal lobe, which is important for cognition in experts[14]. and needed for prediction and observation of behaviors[15], and that experts and non-experts have different neuronal mechanisms[16].

This study was aimed to suggest a model of brain-based experience-knowledge in order to improve dentist's decision making process. In order to decrease problems in decision making that can exert significant influences, this study was developed a model of dentists' experiential knowledge in decision making in order to improve dental experts' decision-making process. Dentists' EEG characteristics, which influence complex clinical decision making processes, were analyzed, and a brain-based learning model to improve experts' clinical decision making was proposed based on this. 


\section{Methods}

In this study, dental experts were defined as those who have been continuously working in clinical dentistry for 10 years or more and can effectively solve problems in clinical decision making. This study was performed in the following two projects in order to develop a brain-based model of experts' experiential knowledge: (1) The first study is a qualitative study of clinical decision making in a clinical scenario. (2) The second study is an analysis of differences in cortical activity of experts and novices through EEG.

In the 1st study, in order to investigate the thought processes used for decision making performed in a real-life clinical setting, the setting was videotaped. While reviewing the videos together, the participants' characteristics in thought processes for decision making were deduced through the think-aloud method and focus group analysis. In the 20th century, the Wurzburg group of philosophers developed this into a new experimental method termed 'introspection' in associative theories that explain human mental processes using idea and association. Initial experiments involved free association of words and explanation of the thought processes $[17,18]$. And then in order to supplement the think-aloud method, this study used focus group analysis to analyze the decision making process in dental experts, which was the focus of this study, in more detail.

In this study, a standardized patient was recruited to reproduce clinical situations and developed a scenario for the patient based on her symptoms. This patient's case was standardized, and all participants participated in an experiment on their decision making process. The scenario for clinical decision making (standardized patient) is are following. "Wisdom Teeth Problem: The patient has been feeling discomfort in upper right molar since two years ago, and food sometimes gets stuck at the location after eating. With recent increases in stress from work, the symptoms at the site have worsened, and the patient feels throbbing pain".

In the 2nd study, the three major steps established through the experiment conducted under an environment simulating the actual clinical environment were developed further into tasks to measure brain waves in the dental experts and novices. 'Confirmation of the patient's chief complaints', 'oral examination', and 'radiography reading' steps were developed into six tasks, and the experts' and novices' cortical activities were measured through a 32-channel EEG. The data measured during the six tasks were pre-processed to statistically analyze the differences between the groups.

Two sets consisting of one piece of auditory information and two pieces of visual information are presented while EEG signals are measured from each participant. Set 1 involves stimuli about an identical patient whereas set 2 involves stimuli about different patients. Here, the expert and novice dentists were asked to record, with a stopwatch, the time required for them to identify the task, identify the patient's problem, and make a decision, in order to analyze differences in the timing of diagnosis at which a decision was made. Figure 1 shows the overall experimental procedure.

(Fig. 1) 
This study was used Matlab and EEGlab for noise filtering and data analysis. Data reprocessing were preprocessed to analyze the changes in EEG signals on the scalp by analyzing the differences between the two groups in terms of phase distribution of each frequency range (beta, alpha, theta, and delta bands) using EEG lab. The data Preprocessing(continuing EEG) are following: Data reduction(ex, Bad channels selection) $\diamond$ Re-reference $\diamond$ Electrooculogram remove - independent component analysis(ICA) $\diamond$ Filtering $\diamond$ Visual inspection.

Using Band Pass Filter by FFT, waves with frequencies above $50 \mathrm{~Hz}$ were removed. This is FFT, which is often used to convert the time domain into the frequency domain, and the following Fig. 2's equation is used[19].

(Fig. 2)

For each task, this study was comparatively analyzed changes in frequencies in the expert and novice dentists' decision making (diagnosis). The pre-processed data were tested for significance on IBM SPSS statistics (Version 22.0) for each task in independent samples t-tests and one-way analysis of variance (ANOVA). Using EEGlab, the distribution of activated cortical areas during each task for the experts and novices were comparatively analyzed.

\section{Results}

Using the basic protocol for analysis of differences between novices and experts in each step, the transcripts of think-aloud and in-depth interviews conducted for 10 novices and 10 experts were analyzed through thematic analysis. Figure 3 shows the characteristics in thought processes in clinical decision making in each step in experts and novices.

(Fig. 3)

The dental experts collect and interpret verbal and non-verbal information while thinking about communication from the moment they identify the patient's chief complaints. In step 1, they establish and categorize all possible hypotheses based on experiential knowledge and evidence at the moment they identify the patient's problem. At the same time, they perform backward reasoning of the hypotheses. Here, the dental experts identify facts at each step while maintaining an open mind and keeping in mind that the hypotheses might be different from the actual problem, unlike the novices. Moreover, clinical decision in identifying the chief complaints, diagnosis, and treatment planning is performed in a simultaneous manner rather than in a stepwise manner. The experts move flexibly through all stages whenever they collect new information. Rather than organizing thoughts in the last diagnosis step, the decision making proceeds while the dentists converge and diverge simultaneously at each moment. However, also in this process, the dentists make decisions closer to the definitive diagnosis during oral examination than during the chief complaints step and also while confirming radiographs than during oral examination. 
In contrast, the dental novices focus on identifying facts and collecting information while identifying the patient's chief complaints. The novices tended to think in a stepwise manner from testing until diagnosis. Although some novices established and categorized hypotheses based on the materials learned in classroom and their experiences when they identified the patient's problem in Step 1, most tended to focus on documenting the patient's story. Here, the novices acted and thought according to the learned protocols and failed to think flexibly about other possibilities. The novices tended to select and establish hypotheses during oral examination rather than while identifying the patient's chief complaints. Then, they condensed the hypotheses while confirming the radiographs to make decisions. Moreover, when problems arose, they tended to depend on other experts and to think anew rather than establishing another hypothesis and approaching the problem themselves.

In order to analyze the characteristics during clinical decision making in the brain, cortical activities were analyzed in 10 dentists as experts and 10 dentists as novices. As a result, the two groups did not differ in the background signals measured in a relaxed state with the eyes closed and during the first task (confirmation of the patient's chief complaints), the novices had higher activities in the left frontal lobe, occipital lobe, and parietal lobe than the experts at the steps where oral examination \& radiography reading. After EEG measurement, the accuracy of the participants' diagnoses made to investigate their clinical decision making was analyzed in order to measure the difficulty of each task. In task 6 (oral examination data), which was difficult and had the lowest accuracy of diagnoses made by the experts, the brain activities in both groups were higher than in other tasks; in particular, the experts' activities in left frontal lobe, occipital lobe, and parietal lobe were higher than those observed in the novices.

Two sets of tasks were performed for measurement of EEG signals. Set 1 was presented in the order of auditory data, oral photograph, and radiograph of the same patient whereas set 2 was presented in the order of auditory data, oral photograph, and radiograph of three different patients for whom different decisions were required. When differences in tasks within sets and differences between groups and sets were investigated, no statistical significance was noted. However, this study was identified changes in the EEG signals activated for identical stimuli and those activated for different stimuli in each set in a chronological order, as shown in Fig. 4.

(Fig. 4)

The results may be interpreted differently depending on the difficulty of the tasks. For each task, the participants were given time after EEG signal measurement to document with hands the decision making process used to solve the patient's problem. In this study, the difficulty of tasks was assessed by statistically analyzing the accuracy of diagnoses made by the experts and novices after measuring EEG signals during clinical decision making. Table 1 demonstrates the groups' differences. 
Table 1

The difficulty of tasks used for EEG measurement

\begin{tabular}{|lllllll|}
\hline Task & \multicolumn{2}{l}{ Average } & SD & \multicolumn{2}{c|}{ t } & p \\
\cline { 2 - 5 } & Expert & Novice & Expert & Novice & & \\
\hline Task1 & 1.00 & .90 & .000 & .316 & 1.000 & .331 \\
\hline Task2 & 1.00 & .80 & .000 & .422 & 1.500 & .151 \\
\hline Task3 & 1.00 & .70 & .000 & .483 & 1.964 & .065 \\
\hline Task4 & 1.00 & .10 & .000 & .316 & 9.000 & $.000^{\star}$ \\
\hline Task5 & .80 & .20 & .422 & .422 & 3.182 & $.005^{\star}$ \\
\hline Task6 & .40 & .00 & .516 & .000 & 2.449 & $.037^{\star}$ \\
\hline
\end{tabular}

(Table 1)

Since this task was more difficult than others, the brain areas would have been activated in the experts to proceed with decision making.

\section{Discussion}

This study aims to analyze the differences in clinical decision making and problem solving between dental experts and novices and to develop tasks based on processes with significant differences. This study was also aimed to measure EEG signals from the participants and to analyze the characteristics of brain activation in experts, in an attempt to explore brain-based knowledge models used by experts in clinical decision making. This study obtained the following conclusions.

As with Wainwright, who suggested that expert clinical decision making is based on wide, organized knowledge, this study was also confirmed that clinical problem solving in experts proceeds based on evidence under a wide range of thinking[20]. However, in contrast to the report that most dental experts make clinical decisions based on forward reasoning, this study was observed backward reasoning. The dental experts still differed from the novices in that they use wide, organized knowledge to make decisions. The experts and novices showed differences in their decision making in the clinical treatment experiment in history taking, oral examination, and radiography reading stages, so these were selected as the basic frames for tasks in ERP to analyze the differences in brain activation between the experts and novices. When differences in cortical activation during clinical decision making were compared between the expert and novice dentists, this study was observed different types of differences. We also confirmed the results of Shallice who suggested that experts have activation in the middle frontal lobe, those of Nichelli et al. who suggested that the prefrontal and parietal lobes are important in experts when compared to novices[21, 22, 23]. Moreover, our results supported other previous studies that performed EEG analysis in chess experts and found that the activation of right frontal, occipital, and parietal lobes is 
more prominent compared to the left hemisphere[24, 25, 26, 27]. This study also found that the activation of EEG signals in the experts was relatively higher when compared to the novices in task 6 , which had the highest difficulty for the dental experts, indicating that the experts also had different cortical activation based on the difficulty of tasks. As suggested by Duncun, Seitz, Kolodny et al. and Chen et al., both hemispheres were activated when performing higher-order cognitive functions although the activities differed depending on the tasks being performed[28, 29, 30, 31].

Maclean suggested the tribune brain theory and developed a model of brain-based teaching of subjects, and studies have investigated its effects[32]. This study could also associate the function of cortical areas activated during dental experts' decision making with teaching and learning. Here, this study is suggested learning directions for brain-based clinical decision making that can be approached to raise experts in dentistry based on brain use. First, a clinical decision making training should be provided in which the dentist can perform backward reasoning and maintain an open mind to other possibilities while recognizing certain information of the patient. Second, Sousa reported that students with different sensory preference react differently to identical stimuli[33]. Trainings should discourage the use of certain brain areas and rather present various learning stimuli and learning formats to enable the use of both brains. Third, by developing education models that present various patient cases and focus on clinical expressions, stimuli from patient experiences should be maximized and dentists should be encouraged to accumulate and integrate experiential knowledge. Fourth, in order to further activate the parietal lobe, which is responsible for somatosensation and higher-order sensory processing, opportunities to experience more multidimensional tasks than the currently performed tasks should be provided. Fifth, to activate both brains, instructional methods that can equally activate the left brain focusing on logical thinking and the right brain focusing on intuitive thinking should be developed and applied.

\section{Conclusion}

This study analyzed differences between dental experts and novices in problem solving and EEG signals during clinical decision making and explored a model of clinical decision making based on brain-based knowledge used by experts in clinical decision making. Based on the findings, this study developed and suggested a model of decision making process in expert and novice dentists, and suggested the basic directions for brain-based learning needed to raise experts based on brain activity. This model is expected to serve as findings of basic brain-based research that investigated steps and factors to improve core competency required for dentists' clinical decision making and suggested ways to improve accurate clinical decision making capabilities with little time and effort. Because of limits in methodology, this study could not be conducted on a large number of participants. In order to generalize the differences in clinical decision making between experts and novices found in this study, bigger samples should be used, and studies should continue with participants of various backgrounds.

\section{Abbreviations}


Electroencephalography

ICA

Independent component analysis

FFT

Fast Fourier Transform

\section{Declarations}

\section{Ethics approval and consent to participate}

This study was approved by the Seoul National University Dental Hospital, Institutional Review Board(IRB No. CRI17006). We obtained the written consent of study participants clearly.

\section{Consent for publication}

Not applicable.

\section{Availability of data and material}

The datasets used in this study are stored at the XXXXXX University, School of Dentistry in Korea. They are available from the corresponding author on reasonable request.

\section{Competing interests}

The authors declare that they have no competing interests.

\section{Funding}

This research was supported by the Research Affairs of the Seoul National University Fundation (No. 86020160023).

\section{Authors' contributions}

YJ contributed to conception, design, acquisition, analysis, interpretation, drafted manuscript, critically revised manuscript. HK contributed to design, analysis, and critically revised manuscript. SB contributed to conception, acquisition and critically revised manuscript. JL contributed to design, interpretation, drafted manuscript and critically revised manuscript. All authors gave their final approval and agree to be accountable for all aspects of the work.

\section{Acknowledgements}


Not applicable.

\section{References}

1. Ericsson KA, Lehmann AC. Expert and exceptional performance: Evidence of maximal adaptation to task constraints. Annual review of psychology. 1996;47(1):273-305.

2. Chi MT, Koeske RD. Network representation of a child's dinosaur knowledge. Developmental psychology. 1983;19(1):29.

3. Ericsson KA, Charness N. Cognitive and developmental factors in expert performance. In: Feltovich PJ, Ford KM, Hoffman RR (editors. ), Expertise in context. Mahwah: Erlbaum; 1997.

4. Ericsson KA. Creative expertise as superior reproducible performance: Innovative and flexible aspects of expert performance. Psychological Inquiry. 1999;10(4):329-33.

5. Johnson RR, Stone BT, Miranda CM, Vila B, James L, James SM, Berka C. Identifying psychophysiological indices of expert vs. novice performance in deadly force judgment and decision making. Frontiers in human neuroscience. 2014;8:512.

6. Holyoak KJ. 12 Symbolic connectionism: toward third-generation theories of expertise. Toward a general theory of expertise: Prospects and limits. 301. 1991.

7. Herling RW. Expertise: The development of an operational definition for human resource development. In Academy of human resource development. 1998;715 - 22.

8. Edmonds E, Candy L, Slatter P, Lunn S. Issues in the design of expert systems for business. In Expert systems human issues. MIT Press. 1990.

9. Piajet J. Play, Dreams, and Limitation in Children. Norton. 1962.

10. Chi MT, Feltovich PJ, Glaser R. Categorization and representation of physics problems by experts and novices. Cognitive science. 1981;5(2):121-52.

11. Baumann A, Deber R. The limits of decision analysis for rapid decision making in ICU nursing. J Nurs Sch. 1989;21(2):69-71.

12. Nakata $H$, Yoshie M, Miura A, Kudo K. Characteristics of the athletes' brain: evidence from neurophysiology and neuroimaging. Brain research reviews. 2010;62(2):197-211.

13. Gutierrez-Davila M, Rojas FJ, Antonio R, Navarro E. Response timing in the lunge and target change in elite versus medium-level fencers. European Journal of Sport Science. 2011;1-8.

14. Luo ZQ, Ma WK, So AMC, Ye Y, Zhang S. Semidefinite relaxation of quadratic optimization problems. IEEE Signal Process Mag. 2010;27(3):20-34.

15. Abernethy B, Neal RJ, Koning P. Visual-perceptual and cognitive differences between expert, intermediate, and novice snooker players. Applied cognitive psychology. 1994;8(3):185-211.

16. Chang K, Chen W. L-cysteine-assisted synthesis of layered MoS2/graphene composites with excellent electrochemical performances for lithium ion batteries. ACS nano. 2011;5(6):4720-8.

17. Mackey A, Gass SM. Stimulated recall methodology in second language research. Routledge. 2013. 
18. Ericsson KA. Protocol analysis and expert thought: Concurrent verbalizations of thinking during experts' performance on representative tasks. The Cambridge handbook of expertise and expert performance. 2006;223 - 41..

19. Harris CM. The Fourier analysis of biological transients. J Neurosci Methods. 1998;83(1):15-34.

20. Wainwright SF, Shepard KF, Harman LB, Stephens J. Factors that influence the clinical decision making of novice and experienced physical therapists. Physical Therapy. 2011;91(1):87-101.

21. Hudspith MJ. Glutamate: a role in normal brain function, anaesthesia, analgesia and CNS injury. British journal of anaesthesia. 1997;78(6):731-47.

22. Nichelli P, Grafman J, Pietrini P, Alway D, Carton JC, Miletich R. Brain activity in chess playing. Nature. 1994;369(6477):191.

23. Shallice T. The Fractionation of Supervisory Control. The cognitive neurosciences. Cambridge: MIT Press; 2004.

24. Allen G, Buxton RB, Wong EC, Courchesne E. Attentional activation of the cerebellum independent of motor involvement. Science. 1997;275(5308):1940-3.

25. Andreasen NC, O'Leary DS, Paradiso S, Cizadlo T, Arndt S, Watkins GL, Hichwa RD. The cerebellum plays a role in conscious episodic memory retrieval. Human brain mapping. 1999;8(4):226-34.

26. Kim SG, Ugurbil K, Strick PL. Activation of a cerebellar output nucleus during cognitive processing. Science. 1994;265(5174):949-51.

27. Farrer $C$, Frith $C D$. Experiencing oneself vs another person as being the cause of an action: the neural correlates of the experience of agency. Neuroimage. 2002;15(3):596-603.

28. Duncan J, Seitz RJ, Kolodny J, Bor D, Herzog H, Ahmed A, Emslie H. A neural basis for general intelligence. Science. 2000;289(5478):457-60.

29. Chen X, Zhang D, Zhang X. A functional MRI study of highlevel cognition: II. The game of GO, Brain Res. Cogn Brain Res. 2003;16:32-7.

30. Jeong JB, Yoon SA. Psychological Factors Related to the Attentional Control of Pro-Gamer. The Korean Society for Computer Game. 2008;14:217-23.

31. Haslinger B, Erhard P, Altenmüller E, Hennenlotter A, Schwaiger M, Gräfın von Einsiedel H, CeballosBaumann AO. Reduced recruitment of motor association areas during bimanual coordination in concert pianists. Human brain mapping. 2004;22(3):206-15.

32. MacLean PD. The triune brain in evolution: Role in paleocerebral functions. Springer Science \& Business Media. 1990.

33. Soursa DA How the brain learns(2nd ed.). Thousand Oaks, CA: Corwin Press, INC. 2001.

\section{Figures}



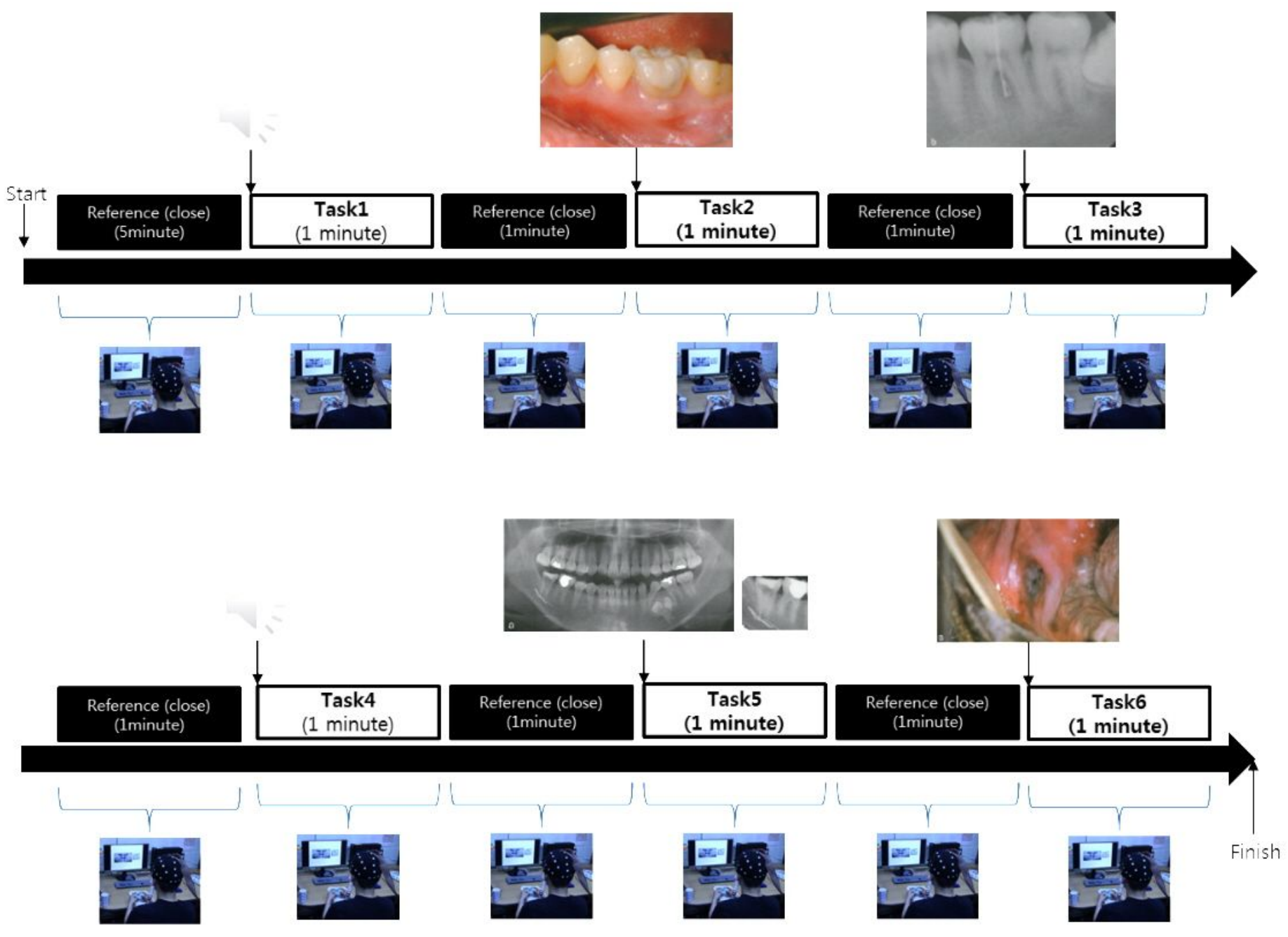

Figure 1

EEG experimental procedure 
1. The $N$-point Discrete Fourier Transform (DFT) can be written as:

$$
X_{N}[k]=\sum_{n=0}^{N-1} x[n] \mathrm{e}^{-j \frac{2 \pi}{N} k n} .
$$

Assume that $N=2, x[0]=1$ and $x[1]=-1$. Find $X_{N}[0]$ and $X_{N}[1]$.

2. The following equation is a Fast Fourier Transform equation:

$$
X_{N}[k]=\sum_{r=0}^{\frac{N}{2}-1} x[2 r] W_{N / 2}^{r k}+W_{N}^{k} \sum_{r=0}^{\frac{N}{2}-1} x[2 r+1] W_{N / 2}^{r k}
$$

where $N$ is the number of samples and $W_{N}=e^{-j \frac{2 \pi}{N}}$. How many complex multiplications are needed in order to calculate $X_{N}[k]$ for $k=0,1, \cdots, N-1$ by using the above equation? Explain your answer.

3. Write the equations (1) and (2) for $N=4$. Show that both equations are equivalent to:

$$
X_{4}[k]=\left(x[0]+x[2] W_{4}^{2 k}\right)+W_{4}^{k}\left(x[1]+x[3] W_{4}^{2 k}\right) .
$$

Which properties of $W_{4}=e^{-j \frac{2 \pi}{4}}$ you have to use in order to show the equivalences?

\section{Figure 2}

Fast four iertrans formation equation 


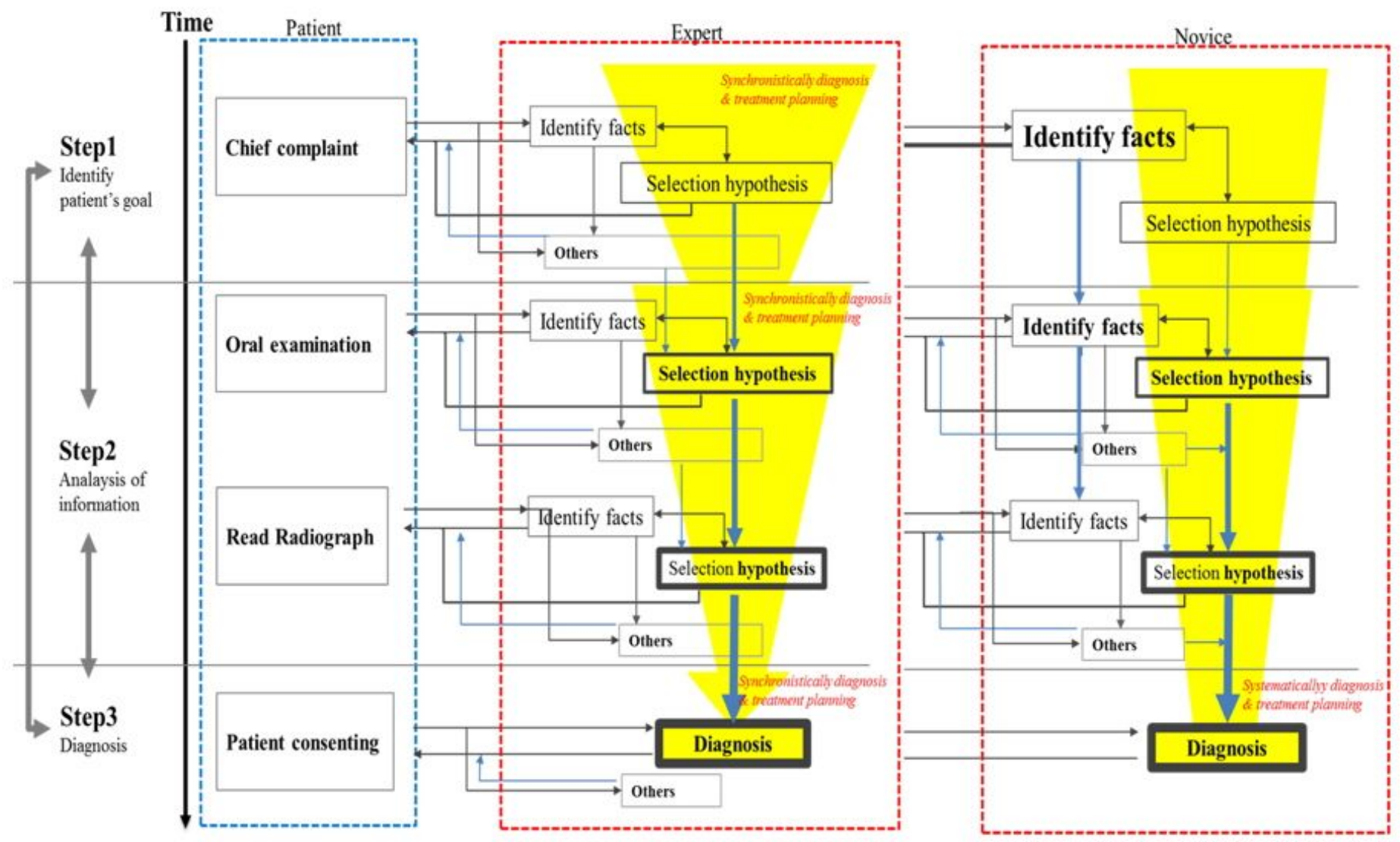

Figure 3

Clinical decision making in expert sandnovices 


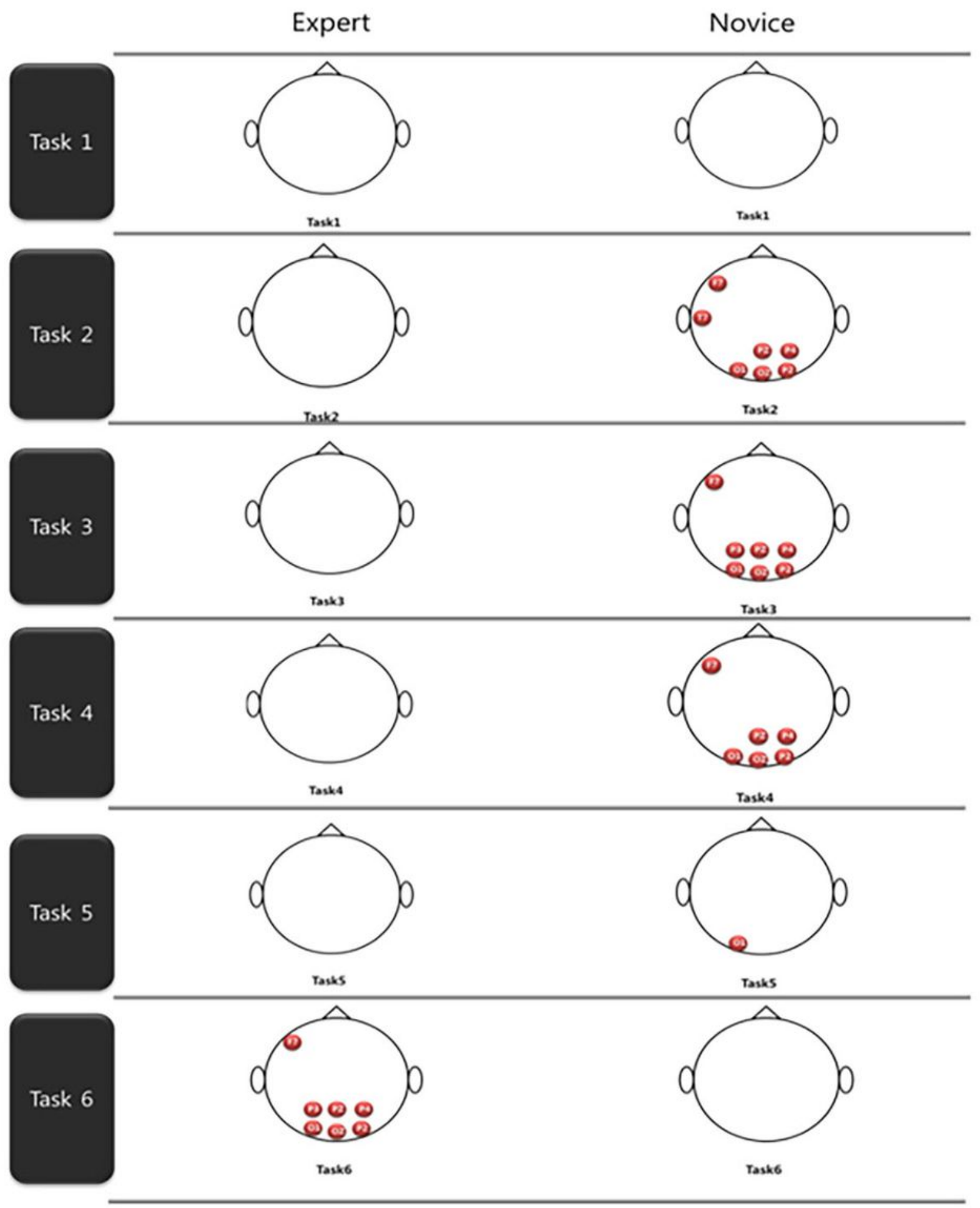

Figure 4

The differences between the experts and the novices 